\title{
Themen
}

Ringo Narewski ${ }^{1}$

\section{Raub- und Beutegut in den Beständen des Bibliothekssystems der Freien Universität Berlin}

Zusammenfassung: Der Aufsatz skizziert die derzeitigen Vorstellungen und Bemühungen der Bibliotheken der Freien Universität Berlin, die Suche nach Raub- und Beutegut in den eigenen Beständen aktiv auf wissenschaftlichem Niveau zu betreiben. Zugleich ist es der Versuch, die Komplexität dieses Unterfangens zu umreißen.

Schlüsselwörter: Universitätsbibliothek <Universitätsbibliothek Berlin, Freie Universität>, Provenienzforschung, Restitution <Kulturpolitik>

\section{Loot in the holdings of the library system "Freie Universität" Berlin}

Abstract: The following article outlines the present conceptions and endeavours of all the libraries of "Freie Universität" in Berlin to locate looted property in their holdings at a professional level. At the same time it is an attempt to give an idea of the complexity of the task at hand.

Keywords: University library, Freie Universität Berlin, Provenance research, Repatriation

Ringo Narewski: narewski@ub.fu-berlin.de

\section{Einleitung}

Im Bibliotheksdienst, Ausgabe 11.2012, erschien unter dem Titel „Erkanntes und Erlebtes" ein Aufsatz über Aussonderung in den Bibliotheken der Freien Universität Berlin. Die beiden Autoren, Karin Andert und Niko Kohls, beide Mitarbeiter

1 Ringo Narewski M.A. ist Referendar an der Universitätsbibliothek der Freien Universität Berlin. 
des Generation Research Program der Ludwig-Maximilians-Universität München, thematisieren darin die Aussonderung einer Ausgabe von Wilhelm Wundts Werk „Erlebtes und Erkanntes“. Dieses Buch enthält, wie die beiden Autoren schreiben, eine Provenienz, welche es als Raubgut ${ }^{2}$ klassifiziert. Es gehörte ursprünglich dem jüdischen Apotheker und Büchersammler Leopold Scheyer, der es 1939 bei seiner Flucht aus Deutschland zurücklassen musste. ${ }^{3}$

Die Aussonderung eines Buches mit einer so deutlichen Provenienz verweist auf Probleme. Die Frage, wie es zu dieser speziellen Aussonderung kam, lässt sich im Nachhinein nicht exakt beantworten. Fehlende Sensibilität gegenüber der Thematik Raub- und Beutegut, zum anderen Unkenntnis der Methoden der Provenienzforschung mögen eine Rolle gespielt haben. Missachtung des Problems von Raub- und Beutegut in den Bibliotheksbeständen durch Bibliothekarinnen und Bibliothekare zu unterstellen, wie es der moralisierende Ton von Andert und Kohls tut, geht jedoch fehl. ${ }^{4}$ Eine solche Pauschalisierung wird nicht nur der Arbeit der Bibliothekare an der Freien Universität Berlin nicht gerecht, sie zeigt ebenso eine allgemeine Unkenntnis der Arbeitsweisen in Bibliotheken.

Der Wert des Artikels besteht dennoch in zwei Punkten. Einmal macht er auf einen Vorgang aufmerksam, der hätte vermieden werden können. Dies gibt Anlass, die eigene Arbeitspraxis zu überprüfen und $\mathrm{zu}$ überdenken. Er bietet Anstoß zu Verbesserungen. Zweitens stellt sich Bibliotheksgeschichte anhand eines Fallbeispiels sehr plastisch dar. Forschung über Raub- und Beutegut und Restitution dient nicht nur der Aufarbeitung von durch Bibliothekare mit verursachtem Unrecht, sondern ist Beitrag zur Institutionengeschichte der Bibliothek und Geschichte eines Berufsstandes. ${ }^{5}$

Der vorliegende Artikel ist als Reaktion auf den Aufsatz von Andert und Kohls zu lesen. Er nimmt die darin enthaltenen Aussagen zum Anlass, das Problem von

2 Im Folgenden wird in Anlehnung an Anton (2010) Raubgut verstanden als „,...] allgemein die nationalsozialistische Entziehung kultureller Güter aus Besitz und Eigentum verfolgter [...] deutscher Staatsbürger innerhalb Deutschlands vor und während des Zweiten Weltkrieges." Davon unterschieden wird der Begriff des Beuteguts. Hierbei handelt es sich um außerhalb Deutschlands durch die Nationalsozialisten entzogene kulturelle Güter. Vgl. Anton, Michael: Illegaler Kulturgüterverkehr. Berlin [u. a.] 2010, S. 404.

3 Vgl. Andert, Karin/Kohls, Niko: Erkanntes und Erlebtes. In: Bibliotheksdienst 45 (2012), H. 11, S. 908-911.

4 Vgl. Andert, Karin/Kohls, Niko 2012, S.909, 911.

5 Vgl. Andert, Karin/Kohls, Niko 2012, S. 911. Babendreier, Jürgen: Erinnerungszeichen. In: Reifenberg, Bernd (Hrsg.): Die Suche nach NS-Raubgut in Bibliotheken. Marburg 2006, S. 8-12, hier S. 9ff. Jahn, Thomas: Die Recherche nach NS-Raubgut aus jüdischem Vorbesitz in der Bayerischen Staatsbibliothek München. In: Reifenberg, Bernd (Hrsg.): Die Suche nach NS-Raubgut in Bibliotheken. Marburg 2006, S. 13-32, hier S. 32. 
Raub- und Beutegut in den Beständen des Bibliothekssystems der Freien Universität Berlin näher zu betrachten.

\section{Raub- und Beutegut in den Bibliotheks- beständen der Freien Universität Berlin}

Die Freie Universität Berlin ist eine Nachkriegsgründung. Als solche erscheint sie erst einmal nicht verdächtig, Raub- und Beutegut in den Beständen ihrer Bibliotheken zu haben. Allein, eine „späte Geburt“ ist kein hinreichender Beweis dafür, dass sich keine durch die Nationalsozialisten zwischen 1933 und 1945 konfiszierte Literatur in den Bibliotheken befindet. Denn es sei ersichtlich, schrieb Ulf Häder von der Koordinierungsstelle für Kulturgutverluste im Jahr 2000, „[...] dass unrechtmäßig entzogene Kulturgüter auch nach 1945 erworben wurden und damit auch Einrichtungen, die 1945 noch nicht existierten, betroffen sein können. “6

Die Entwicklung des Bibliothekssystems der Freien Universität Berlin, besonders das „historische Wachstum“ der Bibliotheksbestände, zeigt exemplarisch, wie Raub- und Beutegut in universitäre Nachkriegsgründungen gelangen konnte. ${ }^{7}$ Als Nachkriegsgründungen konnten die Bibliotheken der Freien Universität Berlin allein mit Rückwärtsergänzungen einen bedeutsamen Bestand an historischer Forschungsliteratur aufbauen. Rückwärtsergänzungen waren nur durch antiquarische Käufe, Schenkungen, Nachlässe, Privatankäufe etc. möglich. Die Bedeutung von Bücherschenkungen in den Anfangsjahren der Freien Universität Berlin verdeutlicht Herma Stamm, Leiterin der Erwerbungsabteilung in der Gründungszeit der Universitätsbibliothek:

„Die Begeisterung über die Neugründung einer Universität in dem Westteil der Stadt Berlin eröffnete ungeahnte Quellen von Spenden, großzügige materielle Unterstützung seitens der amerikanischen Besatzungsbehörden und einen lebhaften Sammeleifer unter allen FU-

\footnotetext{
6 Häder, Ulf: Einführung. In: Beiträge öffentlicher Einrichtungen der Bundesrepublik Deutschland zum Umgang mit Kulturgütern aus ehemaligem jüdischen Besitz. Magdeburg 2000, S. 9-14, hier S. 12.

7 Schnieders, Klaus: Die Erwerbung an der Universitätsbibliothek der FU Berlin. In: Naumann, Ulrich/Fouquet-Plümacher, Doris (Hrsg.): Fünfzig Jahre Universitätsbibliothek der Freien Universität Berlin. Berlin 2002, S. 93-124. Stamm, Herma: Die Anfänge der Universitätsbibliothek der Freien Universität Berlin. In: Naumann, Ulrich/Fouquet-Plümacher, Doris (Hrsg.): Fünfzig Jahre Universitätsbibliothek der Freien Universität Berlin. Berlin 2002, S.3-22, hier S. 3ff. Schlicht, Uwe: Eine politische Geschichte. In: Präsident der Freien Universität (Hrsg.): Freie Universität Berlin. Berlin 1998, S. 9-81, hier S. 10ff.
} 
Angehörigen. Aus dem damals im Ostteil der Stadt noch vorhandenen Bücherreservoir des Buchhandels kauften Studenten und Dozenten gesuchte wissenschaftliche Literatur und schleppten sie in ihre Institutsbibliotheken nach Dahlem.“8

Diese Unterstützung, gerade seitens der amerikanischen Besatzungsbehörden, umfasste nicht nur freiwillige Spenden, sondern auch beschlagnahmte Bücher und Bibliotheken von aufgelösten wissenschaftlichen Einrichtungen der Nationalsozialisten. Klaus Schnieders beschreibt den Bestandsaufbau Ende der 1940er Jahre und in den 1950er Jahren:

„An erster Stelle bei den kostenlosen Erwerbungen zu nennen sind Bestände aufgelöster deutscher Bibliotheken und Spenden amerikanischer Einrichtungen [...]. Eine weitere Quelle der Erwerbungen waren Privatbibliotheken aus beschlagnahmten Häusern. [...] Darüber hinaus gab es weitere Bibliotheken vornehmlich in Dahlem, die auf diesem Wege in die Freie Universität Berlin gelangten. Der größte Teil der Bücher ging an die Fachbibliotheken, der geringere verblieb bei der Bibliothekleitstelle und gelangte schließlich in den Gründungsbestand der Universitätsbibliothek.“9

Es wird deutlich, dass für ein Einbringen von Raub- und Beutegut alle Wege offen standen. Der Umstand, dass große Teile der geraubten und enteigneten Literatur zwischen 1933 und 1945 nach Berlin verbracht wurden, diese einerseits in die Bestände der Berliner Bibliotheken eingingen, andererseits innerhalb des Deutschen Reiches an Bibliotheken weiterverteilt wurden oder in Berlin für eine spätere Verwendung lagerten, schließlich nach 1945 in der völlig zerstörten Stadt oftmals ungesichert vor Zugriffen Interessierter waren, verstärkt diesen Eindruck zusätzlich. Was in dieser Zeit an antiquarischem Schriftgut in Berlin zu kaufen war, stammte zum Teil aus diesen ungesicherten und verwaisten Beständen. ${ }^{10}$ Ein Beispiel hierfür ist die Bibliothek des Berliner Rätekommunisten Alfred Weiland, welche 1979 von der Universitätsbibliothek der Freien Universität Berlin erworben wurde. Ein Teil seiner Sammlung stammt nachweislich aus solchen ungesicherten Beständen. ${ }^{11}$

8 Stamm 2002, S. 4.

9 Schnieders 2002, S. $94 \mathrm{f}$.

10 Vgl. Nitzsche, Grit: Methodische Fragen bei der Recherche nach NS-verfolgungsbedingt entzogenem Kulturgut. In: Regionalverband Berlin-Brandenburg des Vereins Deutscher Bibliothekare (Hrsg.): Raubgut in Berliner Bibliotheken. Berlin 2007, S. 8-13, hier S. 8. Schroll, Heike: Die Bergungsstelle für wissenschaftliche Bibliotheken und andere Quellen zur Provenienzforschung der Berliner Bibliotheken im Landesarchiv Berlin. In: Regionalverband Berlin-Brandenburg des Vereins Deutscher Bibliothekare (Hrsg.): Raubgut in Berliner Bibliotheken. Berlin 2007, S. 18-38, hier S. 19-35.

11 Vgl. Kubina, Sylvia: Die Bibliothek des Berliner Rätekommunisten Alfred Weiland (19061978). Berlin 1995, S. $22 f$. 
Wenn schließlich noch mit bedacht wird, dass die Bemühungen um eine systematische Aufarbeitung der Enteignungen und des Raubs von Kulturgütern in der Bundesrepublik Deutschland meist erst 1998 nach der Unterzeichnung der Washington Conference Principles On Nazi-Confiscated Art ${ }^{12}$ durch die damalige Bundesregierung langsam einsetzten, zeigt sich, dass geraubte und enteignete Bücher jahrzehntelang über sämtliche denkbare Zugangswege, Kauf, Tausch, Schenkung, Nachlass, Leihgabe etc., in die Bestände von Bibliotheken gelangen konnten (siehe Abb. 1). ${ }^{13}$

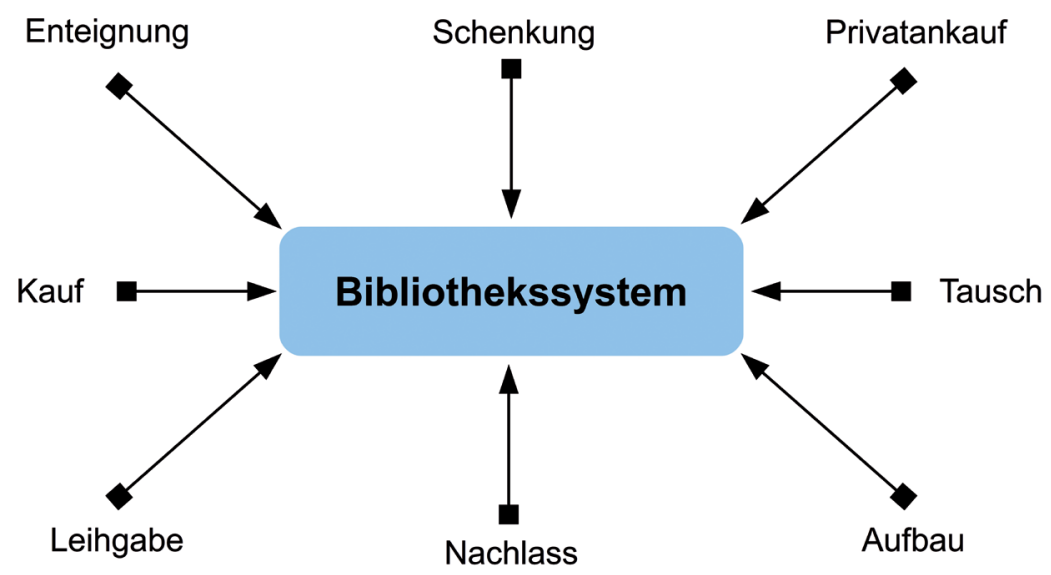

Abb. 1: Zugangswege enteigneter und geraubter Medien.

Während der 1960er bis in die Mitte der 1980er Jahre ist der Bestandsaufbau der Bibliotheken der Freien Universität Berlin durch systematische antiquarische

12 Grundsätze der Washingtoner Konferenz in Bezug auf Kunstwerke, die von den Nationalsozialisten beschlagnahmt wurden: Die Washington Conference Principles On Nazi-Confiscated Art legen für die Rückgabe von NS-verfolgungsbedingt entzogenem Raub- und Beutegut nahe, für mögliche Rückgaben eine ,just and fair solution“ Lösung anzustreben. Vgl. Bindenagel, J. D. (Hrsg.): Washington Conference Principles on nazi-confiscated art. 1998, abrufbar unter: http:// fcit.usf.edu/holocaust/resource/assets/princ.htm, letzter Zugriff am 11.3.2013.

13 Albrink, Veronica/Reifenberg, Bernd: Eine Umfrage unter deutschen Bibliotheken zum Thema „Raubgut“. In: Dehnel, Regine (Hrsg.): Jüdischer Buchbesitz als Raubgut. Frankfurt a. M. 2006, S. 265-276, hier S. 267f. Komorowski, Manfred: Wissenschaftliche Bibliotheken in der NSZeit. In: Kuttner, Sven/Reifenberg, Bernd (Hrsg.): Das bibliothekarische Gedächtnis. Marburg 2004, S. 54-83. 
Erwerbungen gekennzeichnet. So schreibt Stamm etwa in Bezug auf den Sammelschwerpunkt Historische Sozialismusforschung der Universitätsbibliothek, dass „[...] alles auf dem Antiquariatsmarkt Erreichbare, was zum Thema gehörte, [...] sobald der Erwerbungsetat [ab 1966, NR] dies gestattete, dazugekauft“ wurde. ${ }^{14}$ Dies ergibt ein zweites Zeitfenster, in welchem Raub- und Beutegut in größerem Umfang in die Bibliotheksbestände der Freien Universität Berlin gelangen konnte. Bis heute gibt es zudem für das Bibliothekssystem der Freien Universität Berlin keine einheitliche Regelung für die Erwerbung von Antiquaria, die jene Einfallstore für enteignete und geraubte Bücher schließen würde.

Seit geraumer Zeit ist bekannt, dass sich in den Bibliotheksbeständen der Freien Universität Berlin Raub- und Beutegut befindet. Bereits in den siebziger Jahren des vorigen Jahrhunderts wurde von der Bibliothek des Botanischen Gartens und des Botanischen Museums Berlin-Dahlem ein Versuch unternommen, 500 aus Weißrussland stammende Bände $\mathrm{zu}$ restituieren. Ihre endgültige Rückgabe gelang jedoch erst 2010. ${ }^{15}$

In einem anderen Fall, der Bibliothek des Rätekommunisten Alfred Weiland, handelt es sich zu einem guten Teil um Raubgut. Bereits Ende der 1980er und Anfang der 1990er Jahre wurde im hauseigenen Blatt der Universitätsbibliothek der Freien Universität Berlin eine Artikelreihe von H. D. Heilmann veröffentlicht, die sich mit der Herkunft der Bücher in Weilands Bibliothek beschäftigte. ${ }^{16}$ Einige Bände aus dieser sich in der Universitätsbibliothek befindlichen Sammlung konnten von Mitarbeitern und Mitarbeiterinnen zwischenzeitlich ihren heutigen Eigentümern zurückgegeben werden.

Obwohl also seit den 1970er Jahren zweifelsfrei Raub- und Beutegut ${ }^{17}$ in den Beständen der Universitätsbibliothek der Freien Universität Berlin nachgewiesen ist, blieb die Restitution bislang auf Zufallsfunde angewiesen. Eine gezielte Suche

14 Vgl. Schnieders 2002, S. 114ff. Stamm 2002, S. 17.

15 Vgl. Hansen, Melanie: Zurück in die Heimat. In: campus.leben (2010), abrufbar unter: http:// www.fu-berlin.de/campusleben/campus/2010/100525_buchrueckgabe_botanischer_garten/ index.html, letzter Zugriff am 6.2.2013.

16 Vgl. Heilmann, H. D.: Die Bibliothek in Zeit und Räumen I. In: Bibliotheks-Informationen (1988), Nr. 18, S. 2-10. Heilmann, H. D.: Die Bibliothek in Zeit und Räumen II. In: BibliotheksInformationen (1992), Nr. 24, S. 7-8. Heilmann, H. D.: Die Bibliothek in Zeit und Räumen III. In: Bibliotheks-Informationen (1993), Nr.25, S.11-13. Heilmann, H. D.: Die Bibliothek in Zeit und Räumen IV. In: Bibliotheks-Informationen (1994), Nr. 26, S. 14-23.

17 Die beiden angeführten Beispiele zeigen, wie wesentlich die Unterscheidung zwischen Raubund Beutegut ist. Bei den Weißrussland zurückgegebenen Bänden handelt es sich im Sinne der oben eingeführten Trennung um Beutegut. Die Bücher aus der Bibliothek von Alfred Weiland sind dagegen Raubgut. 
nach Raub- und Beutegut in den Beständen der Bibliotheken der Freien Universität Berlin fand bislang nicht statt. Seitens der Universitätsbibliothek wurde dabei das Vorhandensein von enteigneten und geraubten Büchern in den Beständen jedoch nie geleugnet, man war sich des Problems bewusst. ${ }^{18}$

Institutionelle Umstrukturierungen in den vergangenen Jahren, insbesondere die Zusammenfassung von Bibliotheken, die damit verbundenen Bestandsabgleichungen und Aussonderungen, fördern gegenwärtig zunehmend Bücher zutage, die aufgrund der in ihnen verzeichneten Provenienzen unter Verdacht stehen, aus Enteignung und Raub zu stammen. Provenienzen wie Außenpolitisches Amt der NSDAP, Zentralbibliothek der Hohen Schule, Deutsches Auslandswissenschaftliches Institut, Bibliothek des Rabbinerseminars Berlin, Stefan von Auspitz, Deutsche Akademie für soziale und pädagogische Frauenarbeit usw. machen es unumgänglich, den Umgang mit Raub- und Beutegut an der Freien Universität Berlin auf ein höheres Niveau zu heben (siehe Abb. 2).

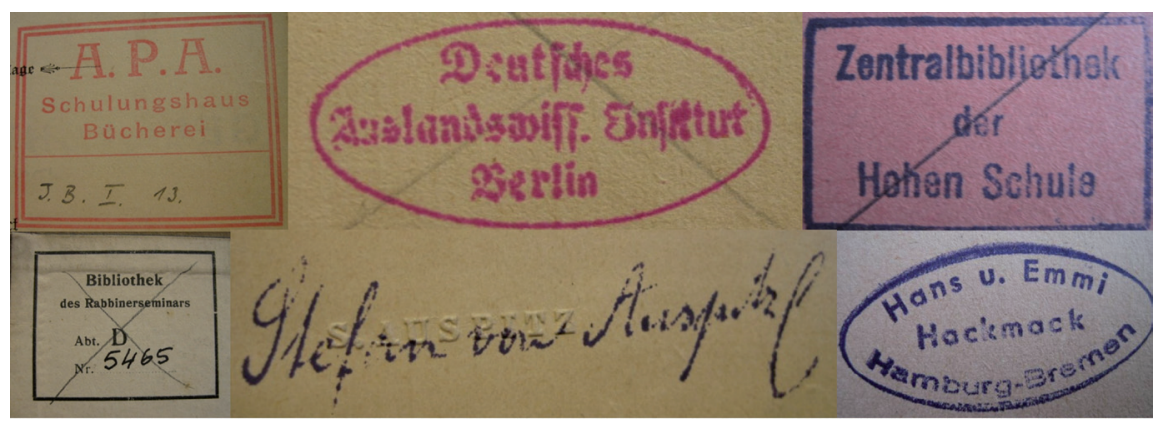

Abb. 2: Provenienzen aus den Beständen des Bibliothekssystems der FU Berlin.

Diese Erkenntnis bestand bereits, als durch Andert und Kohls der „Fall Scheyer/ Wundt“ den Bibliothekaren an der Freien Universität Berlin bekannt wurde. Es gab bereits Problemanalysen mit dem Ziel, die Suche nach Raub- und Beutegut im Bibliothekssystem der Freien Universität Berlin intensiv zu betreiben. Die Aussonderung des Werkes von Wundt bestätigt die Dringlichkeit dieses Vorhabens.

18 Welt Online: In den Bibliotheken lagern Tausende Beute-Bücher. In: Welt Online (2008), abrufbar unter: http://www.welt.de/regionales/berlin/article2444882/In-den-Bibliotheken-lagernTausende-Beute-Buecher.html, letzter Zugriff am 6.2.2013. 


\section{Rahmenbedingungen für die Provenienz- forschung an den Bibliotheksbeständen der Freien Universität Berlin}

Die systematische Suche nach Raub- und Beutegut in Bibliotheksbeständen bedeutet die Durchsicht von tausenden, zehntausenden, oft hunderttausenden von Einzelbänden. Wo eine Bibliothek bereits vor 1945 gegründet wurde, besteht bei der Bewältigung dieser Aufgabe ein gewisses Erleichterungspotenzial, wenn zugleich die Erwerbungsunterlagen aus der Zeit vor dem Ende des Zweiten Weltkrieges erhalten sind. Dann kann bereits anhand dieser Unterlagen bei verdächtigen Buchfunden deren Eingang in die Bibliothek überprüft werden und, soweit vor 1933 in die Bibliothek gelangt, für einen Teil der Funde die Notwendigkeit weiterer Nachforschungen ausgeschlossen werden. Ein zweiter Vorteil betrifft die historische Phase zwischen 1933 und 1945, denn ist in den Zugangsbüchern auch die Herkunft von Beständen, die in dieser Zeit in die Bibliothek gelangten, verzeichnet, bietet sich eine Chance der zweifelsfreien Identifizierung von Raubund Beutegut. ${ }^{19}$

Sind zudem noch Korrespondenzen dieser Zeit zwischen der Bibliothek und ihren Lieferanten erhalten, können auch diese als Hilfsmittel bei der Forschung nach Raub- und Beutegut herangezogen werden. Wo es aber an all dem mangelt, bleibt allein die Durchsicht der Bibliotheksbestände, die vor 1946 erschienen sind. Die Autopsie am Regal, das In-die-Hand-Nehmen tausender und abertausender Bücher, Zeitschriftenhefte und -bände, Karten usw. ist dann die einzig gangbare Methode, um systematisch enteignete und geraubte Medien zu finden. ${ }^{20}$ Für Bibliotheken, die nach 1945 gegründet wurden, ist dies oftmals der alleinige Weg,

19 Vgl. Pudler, Heike: Geschäftsgänge zur Restitution von NS-Raubgut in der Staatsbibliothek zu Berlin. In: Bödeker, Hans Erich/Bötte, Gerd-Josef (Hrsg.): NS-Raubgut, Reichstauschstelle und Preußische Staatsbibliothek. München 2008, S. 147-158, hier S. 149f. Gerlach, Annette: Raubgut in der Zentral- und Landesbibliothek Berlin. In: Regionalverband Berlin-Brandenburg des Vereins Deutscher Bibliothekare (Hrsg.): Raubgut in Berliner Bibliotheken. Berlin 2007, S. 39-49, hier S. 39ff. Über Möglichkeiten und Grenzen der Arbeit mit Zugangsbüchern, Akzessionsjournalen und anderen Erwerbungsunterlagen bei der Suche nach Raub- und Beutegut in Bibliotheken siehe beispielhaft Sydow, Karsten: Die Akzessionsjournale der Preußischen Staatsbibliothek im Hinblick auf NS-Raubgut und die Reichstauschstelle. In: Bödeker, Hans Erich/Bötte, Gerd-Josef (Hrsg.): NS-Raubgut, Reichstauschstelle und Preußische Staatsbibliothek. München 2008, S. 85105. Reifenberg, Bernd: Recherchen in der Universitätsbibliothek. In: Conze, Eckart (Hrsg.): Displaced books. Marburg 2006, S. 8-28, hier S. $12 \mathrm{ff}$.

20 Vgl. Nitzsche 2007, S. 9f. 
Forschung über Raub- und Beutegut in den eigenen Beständen gründlich und nachhaltig zu gestalten.

Was dies bedeuten kann, ist am Beispiel des Bibliothekssystems der Freien Universität Berlin nachvollziehbar (siehe Tab.). Der Gesamtbestand des Bibliothekssystems der Freien Universität Berlin umfasst ca. 8,5 Millionen Bände. Hiervon sind einer Expertenabfrage im OPAC zufolge ca. 499000 Bände vor 1946 erschienen. Diese Bände wären bei einer systematischen Suche nach Raub- und Beutegut durch Autopsie auf Provenienzen hin zu untersuchen. Hinzu kommen bislang nicht elektronisch verzeichnete Bestände, deren genauer Umfang nicht vollständig bestimmbar ist, wohl aber mehrere hunderttausend Bände umfassen dürfte. Dies bedeutet, dass die Zahl der zu überprüfenden Bände stark ansteigt, wobei nicht definierbar ist, wie hoch dieser Anstieg ausfällt. ${ }^{21}$

Diese vorsichtige quantitative Analyse der zu überprüfenden Bestände im Bibliothekssystem der Freien Universität Berlin zeigt bereits, dass Forschung über Raub- und Beutegut hier ein Geschäft von Jahren, womöglich, je nach der Menge und Ausbildung des für eine systematische Suche nach Raub- und Beutegut zur Verfügung stehenden Personals, auch Jahrzehnten ist.

Bibliothek

Trefferzahl Katalog-

Bestände $^{22}$

(nur elektronisch

erfasste Altbestände)

\begin{tabular}{|c|c|c|}
\hline Gesamtes Bibliothekssystem der FU Berlin & 499000 & 8500000 \\
\hline \multicolumn{3}{|c|}{ Detailauswahl einiger Bibliotheken des Bibliothekssystems der FU Berlin } \\
\hline Universitätsbibliothek & 237000 & 2090000 \\
\hline $\begin{array}{l}\text { Bibliothek am Botanischen Garten und } \\
\text { Botanischen Museum }\end{array}$ & 81000 & 201000 \\
\hline Sozialwissenschaftliche Bibliothek & 56000 & 1027000 \\
\hline Philologische Bibliothek & 35500 & 750000 \\
\hline
\end{tabular}

21 Eine interne Schätzung aus dem Jahr 2002 für die Senatsverwaltung für Wissenschaft, Forschung und Kultur Berlin spricht von 2 Millionen zu überprüfender Medieneinheiten. Es heißt im Bericht vom 7.1.2002: „Die geschätzten zwei Millionen sind in den Besitz der Bibliotheken der Freien Universität Berlin durch Schenkungen, Nachlässe, Kauf von Privatbibliotheken, Tausch und antiquarischen Erwerb bei spezialisierten Antiquariaten gelangt.“ Hierbei habe ein Großteil dieser Medien Provenienzen mehrerer Vorbesitzer. Die Kosten einer Überprüfung dieser Bestände wurden mit ca. 2 Millionen Euro veranschlagt. In einer Stellungnahme für die Senatsverwaltung für Wissenschaft, Forschung und Kultur Berlin von 2012 wird eine Schätzung von ca. $130000,-€$ pro Jahr an notwendigen Personalkosten angegeben.

22 Die hier genannten Kennzahlen entsprechen den Selbstdarstellungen der Bibliotheken. 
Bibliothek

Trefferzahl Katalog-

Bestände $^{22}$

abfrage

(nur elektronisch

erfasste Altbestände)

Rechtswissenschaft

31000

800000

Kunsthistorisches Institut/

23000

295000

Bibliothek des Friedrich-Meinecke-Instituts

Bereichsbibliothek Erziehungswissenschaft/

21000

520000

Psychologie

Veterinärmedizin

13000

150000

Geowissenschaftliche Bibliothek

3000

$255000^{23}$

Tab.: Übersicht der größten Bestände an der Freien Universität Berlin mit Erscheinungsjahren vor 1946.

Eine gewisse Erleichterung bei der Suche nach Raub- und Beutegut bietet die fortschreitende elektronische Erfassung der Bestände. Für elektronisch verzeichnete Bestände können Signaturlisten automatisch generiert werden, mittels denen eine gezielte Autopsie am Regal erfolgen kann. Für die nicht elektronisch erfassten Bestände müssten solche Listen erst einmal händisch anhand der alten Zettelkataloge erstellt werden. Haussystematiken, Numerus-currens-, DDCoder RVK-Aufstellungen usw. erschweren eine Durchsicht der nicht elektronisch erfassten Bestände anhand von Signaturgruppen. Da diese Vorgehensweise langwierig ist, sollte mit den Bibliotheken begonnen werden, deren Bestände bereits zum größten Teil elektronisch nachgewiesen sind. Dies bedeutet, die Zeit für sich arbeiten zu lassen, in der Hoffnung, dass der Anteil der nicht elektronisch erfassten Bibliotheksbestände kontinuierlich abnimmt.

Für die bislang nicht elektronisch erfassten Bestände würde es zudem erforderlich werden, einzelne Medien oder ganze Sammlungen, die als Raub- und Beutegut verdächtig sind, bei der elektronischen Erfassung vorzuziehen und gleichzeitig diese Vermutung sichtbar zu dokumentieren. Damit kann verhindert werden, dass solche Zufallsfunde wieder im Büchermeer des Gesamtbestandes versinken. ${ }^{24}$ Wo solche Zufallsfunde gehäuft auftreten, können sie, insofern sie dokumentiert werden, Orientierung geben bei der Suche nach Raub- und Beutegut.

23 Berechnung enthält neben Printmedien auch Karten und Atlanten (Stand 2004).

24 Vgl. Jahn 2006, S. 19. 
Zufallsfunde sind jedoch keine Methode, weshalb sich die Provenienzforschung in den Bibliotheken der Freien Universität Berlin nicht auf diese beschränken kann. Der Satz „Jede Recherche wird scheitern, wenn sie nicht systematisch durchgeführt wird." mag als Aussage zu allgemein klingen, verweist aber darauf, dass ein unsystematisches Vorgehen für einen Erfolg in hohem Maße von Zufälligkeiten abhängig ist. ${ }^{25}$ Zumal die Erfahrung in den Bibliotheken der Freien Universität Berlin zeigt, dass die Häufigkeit solcher Zufallsfunde auch von der Sensibilität und dem Wissensstand der Mitarbeiterinnen und Mitarbeiter in Bezug auf Raub- und Beutegut abhängig ist. Wer nichts sehen will oder nichts über Raub- und Beutegut weiß, wird auch nichts finden.

Die Identifikation von Raub- und Beutegut kann, wie bei solchen Recherchen üblich, nur über ein Ausschlussverfahren erfolgen, indem die in den Medien enthaltenen Vorbesitzervermerke nachverfolgt werden und so ein Wissen über die ursprünglichen Eigentümer aufgebaut wird. Diese Erkenntnisse müssen dokumentiert werden. Zwei Schritte, die Ermittlung von Vorbesitzern und das Auffinden heutiger Eigentümer, stellen hierbei die zeitaufwändigsten Teile der Forschung zu Raub- und Beutegut in den Bibliotheken der Freien Universität Berlin dar. $^{26}$

Während das Auffinden und die erste Dokumentation von Provenienzen nicht zwingend hochspezialisierter Fachkräfte bedarf, sind diese beiden Schritte allein über spezielle Kenntnisse in Recherchetechniken, Schriftkunde, historischen Ereignissen, Institutionengeschichte, Kunstgeschichte und verschiedener Sprachen zu bewältigen. ${ }^{27}$

Das Auffinden und einfache Dokumentieren von Provenienzen kann über die Menge des hierfür zur Verfügung stehenden Personals in seinem Fortschritt stark beeinflusst werden. Die Auseinandersetzung mit den Provenienzen hingegen, erklärt Grit Nitzsche, ,[...] kann [...] nur in Ausnahmefällen von Hilfskräften erledigt werden. [...] hier ist der wachsame Blick des Forschers gefragt, dem Details wie sich wiederholende typische Einbände oder Eintragungen mit einem besonderen Stift nicht entgehen, selbst wenn natürlich nicht jedes Buch von Vorbesitzern kündet.“28

25 Nitzsche 2007, S. 12.

26 Vgl. Nitzsche 2007, S. 9ff. Reifenberg 2006, S. 13ff.

27 Vgl. ebd.

28 Vgl. Nitzsche 2007, S. 10. Ebenso Bödeker, Hans Erich/Bötte, Gerd-Josef: NS-Raubgut, Reichstauschstelle und Preußische Staatsbibliothek. In: Bödeker, Hans Erich/Bötte, Gerd-Josef (Hrsg.): NS-Raubgut, Reichstauschstelle und Preußische Staatsbibliothek, München 2008, S.1-7, hier S. 6. Reifenberg 2006, S. $15 f$. 
Die Identifikation von Vorbesitzern, die zeitliche Reihung der Provenienzen stellt ein weiteres Problem dar, welches Spezialkenntnisse für seine Entschlüsselung verlangt: „In vielen Fällen sind Bücher nach ihrem Raub oder ihrer Beschlagnahmung durch mehrere Hände gegangen, bevor sie an ihrem heutigen Platz ankamen. Sie haben weite Wege zurückgelegt und wurden mit anderen Provenienzen völlig durchmischt.“ Schließlich: „Haben sich Besitzeinträge in Büchern zu Informationen über tatsächliche Personen oder Institutionen gewandelt, beginnt der vierte und z. T. schwierigste Abschnitt der Recherche, die Suche nach heutigen Anspruchsberechtigten.“29

Nitzsche gibt in ihrem Aufsatz zu methodischen Fragen bei der Recherche nach Raub- und Beutegut auch die Schätzung ab, dass ,[...] in tatsächlich geraubten Beständen [...] erfahrungsgemäß bis zu einem Drittel der Bücher mit Provenienzvermerken versehen sind, von denen sich wiederum ca. ein Drittel zur Weiterrecherche eignen." ${ }^{30}$

Jede Bibliothek, deren Geschichte nach 1945 beginnt, steht bei der Ermittlung von Raub- und Beutegut vor einer nicht ohne Weiteres zu nehmenden Hürde. Erwerbungsunterlagen ebenso wie Zugangsbücher taugen nur bedingt zur Recherche, etwa wenn bekannt ist, dass die eigene Institution - wie im Falle der Universitätsbibliothek der Freien Universität Berlin - von Enteignungen nach Ende des Zweiten Weltkrieges profitiert hat. Oder von Verteilungen anderer Art, wie den Zuteilungen in Berlin 1945 und 1946 durch die damals existierende Bergungsstelle für wissenschaftliche Bibliotheken. ${ }^{31}$

Zudem ist die Identifikation und Rückgabe von Raub- und Beutegut gerade in den neugegründeten Bibliotheken der Nachkriegszeit in hohem Maße auf vorhandene Provenienzmerkmale in den Medien selbst angewiesen: „Bibliotheken“, so etwa Bernd Reifenberg, „sind bei [...] Ermittlungen vor einige besondere Probleme gestellt. Das gravierendste besteht darin, dass Bücher in der Regel keine unverwechselbaren Einzelstücke sind, sondern in einer Vielzahl von Exemplaren existieren, von denen nur wenige durch Exlibris, Besitzstempel, handschriftliche Eintragungen, Signaturen, spezielle Einbände etc. eine Art sichtbarer Individualität haben. Fehlen solche individuellen Merkmale, wird die Ermittlung der Vorbesitzer [und damit auch heutiger Eigentümer, NR] von zweifelhaften Zugängen schwierig." 32

29 Nitzsche 2007, S. $11 f$.

30 Nitzsche 2007, S. 10.

31 Vgl. Schroll 2007, S. $18 f f$.

32 Reifenberg, Bernd: Zum Umgang mit NS-Raubgut. In: Kuttner, Sven/Reifenberg, Bernd (Hrsg.): Das bibliothekarische Gedächtnis. Marburg 2004, S. 97-110, hier S. 98. 
Ein Beispiel, das Bibliothekssystem der Freien Universität Berlin betreffend, mag die Schwierigkeiten hierbei nachvollziehbar machen. Das Handbuch historischer Buchbestände verweist in Band 15 darauf: „Bestände der Hochschule für Politik und des Orientalischen Seminars wurden nur vorübergehend aufgenommen und 1948 an die Freie Universität Berlin weitergegeben. “33 Dies ist ein sehr konkreter Hinweis, der einmal genau die Bibliotheken benennt, aus denen mögliche geraubte Literaturbestände in das Bibliothekssystem der Freien Universität Berlin gelangt sein könnten. Zum anderen wird das Jahr der Übergabe an die Freie Universität Berlin genannt. 1948 ist zugleich das Gründungsjahr der Freien Universität Berlin und das Gründungsjahr der Bibliotheksleitstelle, der Vorgängereinrichtung der heutigen Universitätsbibliothek. ${ }^{34}$ Es sollte also möglich sein, diese Bestände in den Zugangsbüchern dieser Zeit aufzuspüren. Letztere dokumentieren die Zugänge jedoch erst ab 1949, als die Bibliotheksleitstelle ihre Arbeit bereits mehrere Monate verrichtete. Obwohl hierin sowohl die Herkunft von Medien wie auch deren Verbleib, ob sie in der Bibliotheksleitstelle verblieben oder an eine der Institutsbibliotheken abgegeben wurden, dokumentiert ist, findet sich kein Hinweis auf besagte Bestände der Bibliothek der Deutschen Hochschule für Politik und der Bibliothek des Seminars für orientalische Sprachen.

Die Suche wird noch dadurch erschwert, dass es sich höchstwahrscheinlich nur um Restbestände dieser Bibliotheken handelte, da der Großteil zu Beginn des Jahres 1946 durch Angehörige der Roten Armee nach Moskau geschafft wurde. ${ }^{35}$ Die Menge dieser Restbestände lässt sich schwer ermitteln. Insgesamt können die Literaturbestände zudem geschlossen in einer Bibliothek verblieben oder an mehrere Bibliotheken des Bibliothekssystems exemplarweise verteilt worden sein. Letzteres ist, wie die Zugangsbücher der Gründungsjahre aufzeigen, eine übliche Praxis gewesen. Literatur wurde dahin gegeben, wo sie gebraucht wurde. Nun könnte noch der Verdacht bestehen, die gesuchten Bestände seien seitens der Freien Universität Berlin an die Deutsche Hochschule für Politik (DHfP) zurückgegeben worden, die sich ebenfalls 1948 neu konstituierte. Doch hierauf findet sich wiederum in den Zugangsbüchern der neugegründeten Bibliothek der DHfP kein Hinweis.

33 Henning, Herzeleide: Bibliothek des Geheimen Staatsarchivs Preußischer Kulturbesitz. In: Krause, Friedhilde (Hrsg.), Handbuch der historischen Buchbestände in Deutschland. Bd.15, Berlin. Hildesheim [u. a.] 1995, S. 54-57, hier S. 55.

34 Vgl. Stamm 2002, S. 4.

35 Lehmann, Klaus-Dieter/Kolasa, Ingo (Hrsg.): Die Trophäenkommissionen der Roten Armee. Frankfurt am Main 1996, Dokumente Nr. 1, Nr. 12, Nr. 31. 
Damit scheitert ein konkreter Hinweis auf Raub- und Beutegut in den Beständen der Bibliotheken der Freien Universität Berlin erst einmal an der fehlenden Dokumentation einer Übergabe von Beständen seitens der damals Zuständigen. Es verwundert zudem, angesichts der Bedeutung der DHfP in der Weimarer Republik und ihres Schicksals zwischen 1933 und 1945, dass in der Geschichtsschreibung dieser Einrichtung nach 1948 kein Hinweis auf die Existenz einer solchen bedeutsamen Sammlung, selbst wenn sie nur noch einen Torso gebildet haben mag, gegeben wird. ${ }^{36}$ Spätestens als Ferhad Ibrahim in den 1980er Jahren seine detailreiche Studie über Die Bibliothek des Fachbereichs Politische Wissenschaft der Freien Universität Berlin schrieb, hätte er bei seinen gründlichen Recherchen auf Hinweise für die Existenz eines solchen Bestandstorsos an der Freien Universität Berlin stoßen müssen. ${ }^{37}$

\section{Derzeitige und geplante Maßnahmen zur Raub- und Beutegutforschung im Bibliotheks- system der Freien Universität Berlin}

Wie kann bei einem geschätzten Bestand zwischen einer halben und zwei Millionen zu prüfender Bände die Suche nach Raub- und Beutegut so gestaltet werden, dass sie nicht wie ein hoffnungsloses Unterfangen angesichts begrenzter Finanz-, Personal- und Zeitressourcen erscheint? Diese Suche und die Restitution möglicher Funde, dies zeigt die reichhaltige Literatur über Provenienzforschung in deutschen Bibliotheken, kann keine Aufgabe nebenbei sein. ${ }^{38}$ Sie lässt sich nicht

36 Vgl. Hornung, Volker/Zehrer, Sabine: Die Bibliothek. In: Greiff, Bodo von (Hrsg.): Das OSI. Berlin 1994, S. 177-188, hier S. 177. Göhler, Gerhard, 1991: Die Wiederbegründung der Deutschen Hochschule für Politik, in: Göhler, Gerhard/Zeuner, Bodo (Hrsg.): Kontinuitäten und Brüche in der deutschen Politikwissenschaft. Baden-Baden 1991, S. 144-164, hier S. 144-151. Suhr, Otto: Die „neue“ Hochschule für Politik. In: Otto-Suhr-Institut (Hrsg.): Das Otto-Suhr-Institut an der Freien Universität Berlin. Berlin 1962, S. 35-48, hier S. 35ff. Köhler, Hans-Werbin: Bibliothek. In: Otto-Suhr-Institut (Hrsg.): Das Otto-Suhr-Institut an der Freien Universität Berlin. Berlin 1962, S. 87. 37 Vgl. Ibrahim, Ferhad: Die Bibliothek des Fachbereichs Politische Wissenschaft (Otto-SuhrInstitut) der Freien Universität Berlin. Köln 1985.

38 Vgl. hierzu die reichhaltige Tagungsliteratur, z. B. Dehnel, Regine (Hrsg.): NS-Raubgut in Museen, Bibliotheken und Archiven. Frankfurt a. M. 2012. Bödeker, Hans Erich/Bötte, Gerd-Josef (Hrsg.): NS-Raubgut, Reichstauschstelle und Preussische Staatsbibliothek. München 2008. Dehnel, Regine (Hrsg.): NS-Raubgut in Bibliotheken. Frankfurt a. M. 2008. Dehnel, Regine (Hrsg.): Jüdischer Buchbesitz als Raubgut. Frankfurt a. M. 2006. 
in dem Sinne in den bibliothekarischen Alltag integrieren, dass sie zu den bisherigen Aufgaben der in Bibliotheken Tätigen einfach hinzugenommen wird. Die Suche nach Raub- und Beutegut in Bibliotheken ist heute, fast ein Dreiviertel Jahrhundert nach den Unrechtstaten der Nationalsozialisten, Forschung in Bibliotheken mit bibliothekarischen Mitteln und Methoden. Provenienzforschung zum Zweck der Auffindung und Restitution von Raub- und Beutegut ist somit Teil der Bibliotheksgeschichte als Gegenstand der Bibliothekswissenschaft. Sie kann nicht von Bibliothekarinnen und Bibliothekaren im Alltagsgeschäft, neben Erwerbung, Katalogisierung, Benutzerservices, Vermittlung von Informationskompetenz, Bestandserhaltung und -pflege, Öffentlichkeitsarbeit usw. mit erledigt werden. Sie ist aufwändig und erfordert ein umfassendes Spezialwissen.

Bibliotheken wie die der Freien Universität Berlin stehen vor der Notwendigkeit einen erheblichen, bislang nur teilweise elektronisch nachgewiesenen Teil ihrer Gesamtbestände durch Autopsie zu überprüfen, wollen sie den Washington Conference Principles On Nazi-Confiscated Art sowie der Erklärung der Bundesregierung, der Länder und der kommunalen Spitzenverbände zur Auffindung und zur Rückgabe NS-verfolgungsbedingt entzogenen Kulturgutes insbesondere aus jüdischem Besitz Folge leisten. Finanz-, Personal- und Zeitaufwand lassen jedoch derzeit die Option einer Überprüfung aller verdächtigen Bestände nur als „theoretisch möglich“ erscheinen. ${ }^{39}$

Dies entbindet nicht von der Verantwortung, sondern nötigt zu einem umsichtigen, bedachten und möglichst effektiven Vorgehen, um dieser Verantwortung soweit wie nur möglich gerecht zu werden. ${ }^{40}$ Immer verbunden mit dem Wissen, sie nicht ganz einlösen zu können. Ziel der Bibliotheken des Bibliothekssystems der Freien Universität Berlin ist es, genau dies zu tun. Darum wurde in die Aussonderungsrichtlinien die Verpflichtung aufgenommen, auszusondernde Medien mit Erscheinungsjahr 1945 oder früher auf Provenienzen hin zu überprüfen. Bei Vorhandensein von Vorbesitzermerkmalen fällt der Universitätsbibliothek die Aufgabe zu, abzuklären, ob es sich bei dem betroffenen Medium möglicherweise um Raub- und Beutegut handelt. Wenn sich der Verdacht erhärtet, sind die notwendigen Schritte für eine Restitution einzuleiten. Hier fehlt bislang jedoch noch der zweite Schritt, eine entsprechende Regelung für zukünftige antiquarische Erwerbungen und Schenkungen.

Zugleich wurde die Notwendigkeit von Informationsstreuung im Sinne der Schaffung eines Problembewusstseins auf Seiten der Bibliotheksmitarbeiterin-

39 Vgl. Jahn 2006, S. $17 \mathrm{f}$.

40 Vgl. Bödeker/Bötte 2008, S. 6. 
nen und -mitarbeiter erkannt. Informationsveranstaltungen zum Thema werden derzeit durchgeführt. Diese stoßen auf reges Interesse seitens der Mitarbeiterinnen und Mitarbeiter. Es zeigte sich bislang, dass ein Problembewusstsein bei vielen Bibliotheksmitarbeiterinnen und -mitarbeitern bereits vorhanden ist. In den bislang geführten Diskussionen ging es nie um die Frage, ob Raub- und Beutegut überhaupt in den Beständen der Bibliotheken der Freien Universität Berlin vorhanden sei. Auch wurde nicht angezweifelt, dass die aufgefundenen Medien als Unrechtsgüter eigentlich nicht in die Bibliothek gehören, sondern den heutigen Eigentümern zurückgegeben werden müssen. Es sind vor allem Fragen danach, wie solcherlei Literatur aufgespürt werden könne, woran sie zu erkennen sei und wie die Aufgabe der Restitution gelöst werden kann, welche die Mitarbeiterinnen und Mitarbeiter beschäftigen. Seit das Problem von Raub- und Beutegut in den Bibliotheksbeständen der Freien Universität Berlin seitens der Universitätsbibliothek aktiv aufgegriffen wurde mit dem Ziel, es auf einer neuen Ebene einer Lösung zuzuführen, zeigt sich eine Bereitschaft vieler Mitarbeiterinnen und Mitarbeiter, hierzu beizutragen.

Es geht letztlich darum, eine systematische Provenienzforschung an den Beständen der Bibliotheken der Freien Universität Berlin in Gang zu setzen. Dies setzt natürlich ein professionelles Wissen seitens der damit befassten Mitarbeiterinnen und Mitarbeiter voraus. Ein Konzept zum Vorgehen ist zu entwickeln. Dabei wird versucht, an die reichhaltigen Erfahrungen von Kollegen und Kolleginnen anderer Bibliotheken anzuknüpfen. Ein intensiver Austausch mit diesen über deren bisherige Arbeit, die angewandten Methoden und Instrumente sowie deren bisherigen Forschungsergebnissen ist gefragt mit dem Ziel, diese alle für die eigene Arbeit fruchtbar $\mathrm{zu}$ machen und gleichzeitig eigene gewonnene Erkenntnisse $\mathrm{zu}$ kommunizieren.

Als nächstes stehen In-house-Schulungen für Bibliotheksmitarbeiterinnen und -mitarbeiter an, in denen Grundkompetenzen im Erkennen und im Umgang mit Medien, die verdächtig sind, Raub- und Beutegut zu sein, vermittelt werden müssen.

Zudem ist eine größere Stichprobe in den Altbeständen geplant, um solidere Aussagen über das Vorhandensein von Raub- und Beutegut in den Bibliotheksbeständen der Freien Universität Berlin zu ermöglichen. Bislang beruht der Großteil an Erkenntnissen auf Zufallsfunden durch aufmerksame Mitarbeiterinnen und Mitarbeiter (sowie Nutzerinnen und Nutzer!). Die Ergebnisse dieser Stichprobe sollen die Grundlage für ein mehrjähriges Provenienzprojekt in der Universitätsbibliothek sein. Für diese Stichprobe bieten sich die Gründungsbestände der Universitätsbibliothek an, also jener Teil, welcher Ende der 1940er bis Ende der 1950er in die Bibliothek gelangte. Hier liegt aufgrund der Bestandsgeschichte die Vermutung nahe, dass darin Raub- und Beutegut enthalten ist. Zudem ist damit 
die Bedingung der Begrenzbarkeit als Grundlage einer Realisierung effektiver Provenienzforschung erfüllt. ${ }^{41}$

Ob es hierdurch langfristig zur notwendigen Institutionalisierung der Suche nach Raub- und Beutegut in den Bibliotheksbeständen der Freien Universität Berlin kommen wird, bleibt abzuwarten. Als Bedingung zur Erfüllung der durch die Bundesregierung, die Bundesländer und die Kommunen an die öffentlichen Bibliotheken adressierten Aufgabe, aktiv nach Raub- und Beutegut in den eigenen Beständen zu suchen und Restitution zu betreiben, wurde dies bereits von anderen Bibliotheken als unumgänglich erkannt. ${ }^{42}$

\section{Schlussbetrachtung}

Der Aufsatz von Andert und Kohls brachte ein Problem zutage. Bislang gab es keine systematischen Versuche seitens des Bibliothekssystems der Freien Universität Berlin, die Forderungen der Washington Conference Principles On Nazi-Confiscated Art und der Erklärung der Bundesregierung, der Länder und der kommunalen Spitzenverbände zur Auffindung und zur Rückgabe NS-verfolgungsbedingt entzogenen Kulturgutes insbesondere aus jüdischem Besitz umzusetzen. Letztlich führte vermutlich das Fehlen eines auf breite Innenwirkung ausgelegten Wissens um Raub- und Beutegut sowie um die Provenienzforschung in deutschen Bibliotheken zur Aussonderung eines Buches mit eindeutiger Provenienz.

Dies ist der Anlass für die hier vorgelegte Skizze über die derzeitigen Vorstellungen und Bemühungen der Bibliotheken der Freien Universität Berlin, die Suche nach Raub- und Beutegut in den eigenen Beständen aktiv auf wissenschaftlichem Niveau zu betreiben. Zugleich ist es der Versuch, die Komplexität dieses Unterfangens zu umreißen.

Es wurde bewusst keine moralisierende Ebene eingenommen, um den Weg für eine umfassende fachliche Diskussion über Möglichkeiten und Grenzen der Restitutionsbemühungen in Bibliotheken offen zu halten. Wenn es dabei zugleich gelungen ist, entgegen der Empörung, die in Anderts und Kohls Aufsatz spürbar wird, zu zeigen, dass weder eine Missachtung des Problems von Raub- und Beutegut in den eigenen Beständen die Haltung der Bibliotheksmitarbeiterinnen und -mitarbeiter an der Freien Universität Berlin prägt, noch, dass von diesen ein

41 Vgl. Jahn 2006, S. 18.

42 Vgl. z.B. http://raubgut.zlb.de/ oder http://staatsbibliothek-berlin.de/die-staatsbibliothek/ abteilungen/historische-drucke/aufgaben-profil/ns-raubgut/, letzter Zugriff am 21.1.2013. 
leichtfertiger Umgang mit dem „Kulturgut Buch“ gepflegt wird, hat dieser Aufsatz aus Sicht seines Autors bereits seinen Zweck erfüllt. Zugleich ist er eine Einladung zum fachlichen Austausch, bevorzugt in konstruktiv-kritischer Natur.

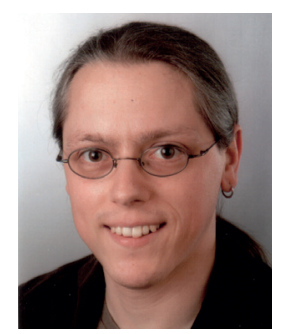

\section{Ringo Narewski}

Universitätsbibliothek der

Freien Universität Berlin

Garystr. 39

14195 Berlin

Email: narewski@ub.fu-berlin.de 